\title{
On the Coupling of Longitudinal and Transverse Waves in a Linear Three-Element Viscoelastic String Subjected to Transverse Impact
}

\author{
Jack C. Smith and Jeffrey T. Fong ${ }^{1}$ \\ Institute for Materials Research, National Bureau of Standards, \\ Washington, D.C. 20234
}

(May 10, 1968)

\begin{abstract}
The problem of wave propagation in a simple viscoelastic string subjected to constant velocity transverse impact is reexamined. An error in an earlier solution by Smith (J. Res. NBS 70B, 257 (1966)) is corrected and an alternate numerical scheme based on the method of characteristics but using an implicit formulation of finite-difference equations is presented. The constitutive equation used is that of a linear viscoelastic model consisting of a spring and Maxwell element in parallel. Results of an illustrative calculation are discussed.
\end{abstract}

Key Words: Characteristics; finite-difference equations; linear viscoelasticity; partial differential equations; transverse impact; wave propagation; waves in strings.

\section{Introduction}

The theoretical problem of wave propagation in a uniform string subjected to oblique impact is well understood if one assumes a dynamic stress-strain relation $T=T(\epsilon)$ for the material, where $T$ denotes the tensile force acting along the string, $\epsilon$, the Lagrangian strain, and there is no explicit dependence of $T$ on the rate of strain.

Several types of mathematical complication can enter into this theory. The first type is due to the nonlinearity of the function $T(\epsilon)$. The theory predicts that:

(a) if the curve $T(\epsilon)$ is concave towards the $\epsilon$ axis, strains of increasing magnitude are propagated with decreasing velocity $c$ given by

$$
c=\sqrt{\frac{1}{m} \frac{d T}{d \epsilon}}
$$

where $m$ denotes the mass of the string per unit of unstrained length, and

(b) if the curve $T(\epsilon)$ contains a portion that is concave towards the $T$ axis the strain distribution is discontinuous, containing a strong shock, or group of strains propagating at the same velocity.

Another type of complication arises when the string is allowed to undergo arbitrary spacial motion. Even though the theory is restricted to one-dimensional strain, the velocity at every point of the string is a three-dimensional vector which can be resolved into tangential and normal components representing longitudinal and transverse motions respectively. Depending on the initial shape of the string and the boundary conditions, the two wave motions may be coupled in

1 N.R.C.-N.B.S. Postdoctoral Resident Research Associate, 1966-68. 
the sense that no simple wave solution for each motion can be found. Two characteristic velocities are involved: different values of strain in the longitudinal wave are propagated at velocity $c$ given by (1), and changes in configuration of the string are propagated at different values of the velocity $P$ given by

$$
P=\sqrt{\frac{1}{m} \frac{T}{1+\epsilon}}
$$

For a detailed discussion of this rate-independent theory as applied to string motion, see Cristescu $[1,2] .^{2}$

The complication in the coupling of the two motions is avoided when one considers an infinitely long string, initially straight and subjected to a constant-velocity transverse impact. Simple wave solutions were reported by Smith, McCrackin, and Schiefer [3], and were recently given in another form by Schultz, Tuschak, and Vicario [4]. Attempts to interpret the results of transverse impact experiments on materials such as nylon and polyester yarns, and strips of natural rubber, based on the rate-independent theory, however, were not completely satisfactory. In particular, two basic predictions of the theory were not verified:

(a) The quasistatic and dynamic stress-strain curves of the nylon and polyester samples tested by Petterson and Stewart [5, 6] and by Smith, Fenstermaker, and Shouse [7], have portions concave towards the tension axis, implying that strong shocks should exist in the strain distributions. Neither group noted the presence of any strong discontinuities in their experimental data.

(b) For each impact velocity, the theory yields a unique relation between the strain and the longitudinal wave velocity $c(\epsilon)$. It is an inherent feature of the rate-independent theory that this unique relation is valid for all impact velocities. Experimental data failed to support this assertion after appropriate consideration was given to measurement uncertainties. For additional discussion, see Smith and Fenstermaker [8].

In order to gain insight for interpreting these effects it is useful to study the behavior under impact conditions of a simple linear viscoelastic model, such as one consisting of a spring in parallel with a Maxwell element. ${ }^{3}$ This model has already been used by Morrison [9] and Smith [10] to study wave propagation in filaments impacted longitudinally. By analogy with results obtained in these theoretical studies, the effects of viscoelasticity on experimentally obtained strain, tension, and particle velocity distributions are better understood. By extending the use of this model to the transverse-impact problem, further enlightenment will result. In a viscoelastic string it is not possible to uncouple the mutual influence of the two types of wave as was done in the rateindependent theory under some restrictive assumptions. Thus one must study theoretically such additional effects of viscoelasticity as changes in the transverse wave profile, changes in the transverse wave propagation velocity, and secondary changes in the strain, tension, and particle velocity distributions.

A detailed analysis of such a problem with a proposed numerical scheme suitable for the calculation of strong discontinuities was published by Smith [11]. Since publication several errors have been found with the consequence that the proposed numerical scheme is not valid, although the basic formulation of the problem and the mathematical method of solution remain applicable. In this paper these errors are corrected and the numerical scheme revised. In addition the problem is discussed from a different viewpoint and an alternate numerical scheme presented. Illustrative numerical calculations are also given.

' Figures in brackets indicate the literature references at the end of this paper.

${ }^{3}$ It should be noted that for three-dimensional problems there are objections to the use of constitutive equations characterizing the behavior of spring and dashpot models. Unless properly formulated, such equations, containing time derivatives of the stress and strain, violate the so-called "principle of material indifference" as stated by Truesdell and Toupin [12]. However, the restrictions imposed by this principle are automatically satisfied in the one-dimensional problem considered here. 


\section{Notation}

The symbols used are the same as those used in reference [11] except for the symbol $K$ representing the spring constant.

$m \quad$ initial density of the string in mass per unit length.

$K \quad$ spring constant of the string in force units.

$\lambda$ dimensionless parameter having a value between 0 and 1 such that $\lambda K$ is the spring constant of the Maxwell element constituting one branch of the viscoelastic model.

$\tau \quad$ relaxation time of the Maxwell element.

$c \quad$ velocity of the longitudinal wavefront as given by eq (1); for the three-element model it is given by $c=\sqrt{K / m}$.

$T$ tension in the string in force units.

$P \quad$ velocity related to the propagation of transverse waves as given by eq (2).

$V \quad$ transverse impact velocity.

$t \quad$ time coordinate.

$x \quad$ Lagrangian space coordinate fixed to the string.

$x^{\prime}, y^{\prime}$ laboratory or observer's coordinates to be referred to as horizontal and vertical coordinates, respectively; the $x$ and $x^{\prime}$ coordinates of the string coincide in the initial unstrained state.

$\xi, \eta \quad$ horizontal and vertical components of displacement of a point $x$ on the string relative to its original position:

$$
x^{\prime}=x+\xi(x, t) ; y^{\prime}=\eta(x, t) .
$$

$u, v$ horizontal and vertical components of velocity in $x^{\prime}, y^{\prime}$ space of a point $x$ on the string:

$$
u=\frac{\partial x^{\prime}}{\partial t}=\frac{\partial \xi}{\partial t} ; v=\frac{\partial y^{\prime}}{\partial t}=\frac{\partial \eta}{\partial t}
$$

$\alpha, \beta$ first partial derivatives of the laboratory coordinates of a point $x$ on the string with respect to the Lagrangian coordinate $x$ :

$$
\alpha=\frac{\partial x^{\prime}}{\partial x}=1+\frac{\partial \xi}{\partial x} ; \beta=\frac{\partial y^{\prime}}{\partial x}=\frac{\partial \eta}{\partial x} .
$$

$d s \quad$ infinitesimal length element of the deformed string:

$$
d s=\sqrt{\left(d x^{\prime}\right)^{2}+\left(d y^{\prime}\right)^{2}}=\sqrt{\alpha^{2}+\beta^{2}} d x .
$$

$\epsilon \quad$ Lagrangian strain at $x$ on the string

$$
\epsilon=\frac{d s-d x}{d x}=\sqrt{\alpha^{2}+\beta^{2}}-1
$$

$\theta \quad$ angle that each infinitesimal length element makes with the horizontal $x^{\prime}$ direction:

$$
\cos \theta=\frac{\partial x^{\prime}}{\partial x} \frac{d x}{d s}=\frac{\alpha}{1+\epsilon} ; \sin \theta=\frac{\beta}{1+\epsilon} .
$$

flow or particle velocity of a point $x$ on the string with respect to an inextensible coordinate coinciding with the string and fixed to it at points in advance of the longitudinal-wave front; that is, the rate of extension of the portion of string between point $x$ and the longitudinalwave front. This velocity satisfies the differential equation: 


$$
d w=d u \cos \theta+d v \sin \theta=\frac{\alpha}{1+\epsilon} d u+\frac{\beta}{1+\epsilon} d v
$$

\section{Formulation}

The viscoelastic model consists of a spring of spring constant $(1-\lambda) K$ in parallel with a Maxwell element of spring constant $\lambda K$ and relaxation time $\tau$. If the quantity $m c^{2}$ is substituted for $K$, the constitutive equation for this model takes the form

$$
m c^{2} \frac{\partial \epsilon}{\partial t}+\frac{(1-\lambda) m c^{2}}{\tau} \epsilon=\frac{\partial T}{\partial t}+\frac{1}{\tau} T
$$

For plane motion the following two equilibrium equations apply:

$$
\begin{aligned}
& m \frac{\partial u}{\partial t}=\frac{\partial}{\partial x}(T \cos \theta)=\frac{\partial}{\partial x}\left[\frac{\alpha T}{1+\epsilon}\right] \\
& m \frac{\partial v}{\partial t}=\frac{\partial}{\partial x}(T \sin \theta)=\frac{\partial}{\partial x}\left[\frac{\beta T}{1+\epsilon}\right]
\end{aligned}
$$

The equations of continuity are

$$
\begin{aligned}
& \frac{\partial u}{\partial x}=\frac{\partial \alpha}{\partial t} \\
& \frac{\partial v}{\partial x}=\frac{\partial \beta}{\partial t} .
\end{aligned}
$$

We shall consider only the waves propagating in the positive $x$ direction. The solution therefore of the set of eqs (7), (9), and (10) through (14) must satisfy the initial conditions: for $t=0, x>0$

$$
u=v=w=\beta=\epsilon=T=0, \alpha=1,
$$

and the boundary conditions: for $t>0, x=0$

$$
u=0, v=V,
$$

and for $t>0, x \rightarrow \infty$

$$
u=v=w=\beta=\epsilon=T=0, \alpha=1 .
$$

In addition to the initial and boundary conditions, certain compatibility conditions also apply. At the longitudinal wave front $\epsilon, w$, and $T$ are discontinuous and must satisfy the jump relations

$$
\begin{aligned}
& {[w]=-c[\epsilon]} \\
& {[T]=-m c[w]}
\end{aligned}
$$

where the bracketed quantities represent jumps in value occasioned by the passage of the wave front at the point under consideration. At the transverse wave front $u, v, \alpha, \beta$, are discontinuous and must satisfy the jump relations

$$
\begin{aligned}
& {[u]=-P[\alpha-1]} \\
& {[v]=-P[\beta] .}
\end{aligned}
$$




\section{Explicit Method of Solution}

The variables $u, v, \alpha, \beta$ can be eliminated from eqs (7), (9), and (11) through (14) to obtain the equations

$$
\begin{aligned}
& \frac{\partial w}{\partial t}=\frac{1}{m} \frac{\partial T}{\partial x} \\
& \frac{\partial w}{\partial x}=\frac{\partial \epsilon}{\partial t} .
\end{aligned}
$$

These equations and the constitutive eq (10) form a set having characteristics given by

$$
\frac{d x}{d t}= \pm c
$$

and

$$
\frac{d x}{d t}=0
$$

Thus, the strain $\epsilon$, tension $T$, and particle velocity $w$ can have discontinuities only along the characteristic curves $x= \pm c t+a$, and $x=b$, in which $a$ and $b$ are constants of integration. In particular, $\epsilon, T$, and $w$ are discontinuous at the longitudinal wave front, but are continuous at the transverse wave front.

The following equations hold along the characteristics: For the " $+c "$ characteristic

$$
m c d w-d T=\left[T-(1-\lambda) m c^{2} \epsilon\right] \frac{d t}{\tau} ;
$$

for the "-c" characteristic

$$
m c d w+d T=-\left[T-(1-\lambda) m c^{2} \epsilon\right] \frac{d t}{\tau}
$$

for the " $x=b$ " characteristic

$$
m c^{2} d \epsilon-d T=\left[T-(1-\lambda) m c^{2} \epsilon\right] \frac{d t}{\tau} .
$$

Equation (28) is the same as the constitutive eq (10).

The values of the strain, tension, and particle velocity at the longitudinal wave front can be found by solving the set of equations (18), (19), and (26). The results are

$$
\begin{gathered}
\epsilon(c t, t)=\epsilon_{0} e^{-(\lambda t / 2 \tau)} \\
T(c t, t)=m c^{2} \epsilon_{0} e^{-(\lambda t / 2 \tau)} \\
w(c t, t)=-c \epsilon_{0} e^{-(\lambda t / 2 \tau)},
\end{gathered}
$$

where $\epsilon_{0}$ is the strain $\epsilon(0,0)$ resulting at the instant of impact. To find the value of $\epsilon_{0}$, the compatibility conditions (20) and (21) are expressed in the form

$$
u=w+P(1+\epsilon-\alpha)
$$




$$
v=-P \beta
$$

in which the values of $u, v, \alpha$, and $\beta$ are understood to be the values obtained just after passage of the transverse wave front, and the continuous quantities $P, \epsilon$, and $w$ are also evaluated at the wave front. Applying the boundary conditions (16) to eqs (18), (32), and (33), and using eq (7), one finds that at the point of impact the relation $V^{2}=P_{0}^{2}\left(1+\epsilon_{0}\right)^{2}-\left[\left(1+\epsilon_{0}\right) P_{0}-c \epsilon_{0}\right]^{2}$ must be satisfied. From eqs (2), (18), and (19), one obtains $P_{0}=c \sqrt{\epsilon_{0} /\left(1+\epsilon_{0}\right)}$ which, when substituted into the relation above, gives

$$
V^{2} / c^{2}=\epsilon_{0}\left(1+\epsilon_{0}\right)-\left[\sqrt{\epsilon_{0}\left(1+\epsilon_{0}\right)}-\epsilon_{0}\right]^{2} .
$$

The value of $\epsilon_{0}$ is obtained by solving eq (34).

If velocity $P$ is defined as in eq (2), it is obvious that eqs (11) through (14) form a set involving $P$ and the dependent variables $u, v, \alpha$, and $\beta$. This set of equations has characteristics given by

$$
\frac{d x}{d t}= \pm P
$$

aııu along these characteristics the following relations hold: For the " $+P$ " characteristic,

$$
\beta(d u-P d \alpha)-\alpha(d v-P d \beta)=0,
$$

and for the "-P" characteristic,

$$
\beta(d u+P d \alpha)-\alpha(d v+P d \beta)=0 .
$$

The solution of the system of equations (7), (9), (26), (27), (28), (36), and (37), is found by numerical integration along the set of characteristics plotted in the Lagrange diagram, figure 1. In this diagram the straight line characteristics are solutions of the equation $d x / d t= \pm c$; the characteristic passing through the origin, and having the equation $x=c t$ corresponds to the discontinuity marking the wave front of the strain, tension, and particle velocity distributions. Thus, in region 0 between this characteristic and the $x$ axis the strain $\epsilon$, tension $T$, and particle velocity $w$ are zero. Along the region 1 side of the characteristic the values of $\epsilon, T$, and $w$ are given by eqs (29), (30), and (31), which may be thought of as boundary conditions.

The curved line passing through the origin represents a solution of the equation $d x / d t=P$. The exact course of this characteristic is not known in advance, as $P$ is a function of $\epsilon$ and $T$. This characteristic corresponds to the front of the wave of transverse motion that propagates along the filament; thus, in region 1 between this characteristic and the characteristic $x=c t$ the velocity $u$ is equivalent to the velocity $w$, the variable $\alpha$ is equal to $1+\epsilon$, and the velocity $v$ and variable $\beta$ are equal to zero. The jump relations (32) and (33) holding along the transverse wave front constitute a second set of boundary conditions. A third set of boundary conditions expressed by eq (16) holds along the time axis.

Calculation of values for $u, v, w, \alpha, \beta, \epsilon, T$ at the mesh points ii: the Lagrangian diagram (using fig. 1 as an example) proceeds systematically in the following steps:

1. Calculate $\epsilon_{0}$ using eq (34).

2. Calculate $w, \epsilon, T$ for mesh points along the characteristic $x=c t$, using eqs (29), (30), and (31).

3. Calculate $w, \alpha, \beta, \epsilon, T$ at point 1 , and $u, v, w, \alpha, \beta, \epsilon, T$ at point 2 using a subroutine to be described later.

4. Calculate $u, v, w, \alpha, \beta, \epsilon, T$ at successive points $3,4,5,6$.

5. Calculate $w, \epsilon, T$ at successive points $a, b, c$, etc.

6. Continue the calculation as indicated. 


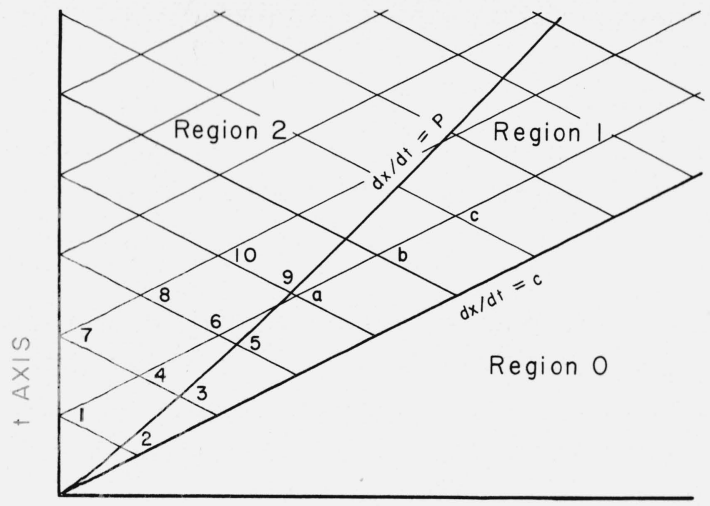

$x$ AXIS

FIGURE 1. Characteristics network used in the explicit method of solution.

The straight line characteristics of positive and negative slope are solutions of the eq $d x / d t= \pm c$. The characteristic separating region 1 from region 0 gives the location of the longitudinal wave front. The curve characteristic separating region 2 from region 1 and satisfying the eq $d x / d t=P$ gives the location of the transverse wave front.

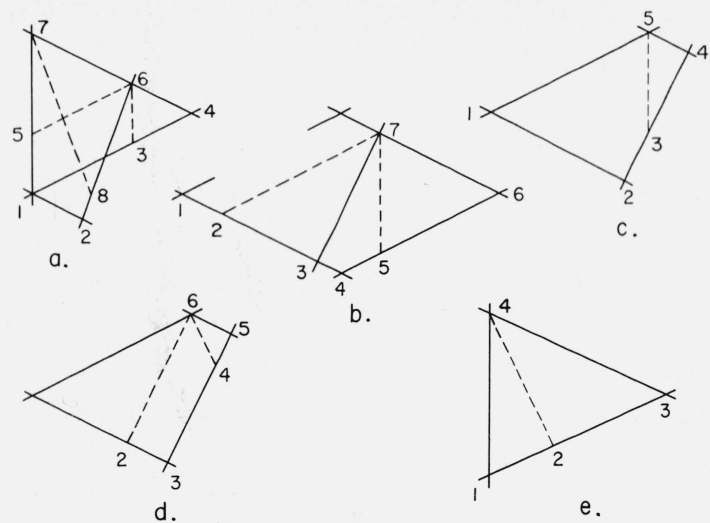

FigURE 2. Mesh situations encountered during integration along the characteristics plotted in figure 1.

Five mesh situations depicted in figure 2 are encountered in the numerical integration process. The simplest of these is that of figure 2c. Here, points 1 and 5 lie at the intersections of $c$ characteristics, and the characteristic between points 2 and 4 may be either a $c$ or a $P$ characteristic; line $(3,5)$ is a constructed vertical line. Values of $w, \epsilon$, and $T$ at point 5 are found from data known at points 1,2 , and 4 by solving eqs (26), (27), and (28) written respectively in the form

$$
\begin{aligned}
& m c w-T=m c w_{1}-T_{1}+\left[T_{15}-(1-\lambda) m c^{2} E_{15}\right]\left(t_{5}-t_{1}\right) / \tau \\
& m c w+T=m c w_{4}+T_{4}-\left[T_{45}-(1-\lambda) m c^{2} E_{45}\right]\left(t_{5}-t_{4}\right) / \tau \\
& m c^{2} \epsilon-T=m c^{2} \epsilon_{3}-T_{3}+\left[T_{35}-(1-\lambda) m c^{2} E_{35}\right]\left(t_{5}-t_{3}\right) / \tau .
\end{aligned}
$$

In these equations the symbol $T_{15}$, for example, represents the average value of $\left(T_{1}+T\right) / 2$. In the first trial calculation $T_{15}$ is set equal to $T_{1}$; the calculation is repeated twice using improved values of $T_{15}$.

Figure 2a depicts a situation found in meshes low on the $t$ axis. In the lowest mesh the transverse wave front characteristic passes through the origin so that points 1 and 2 coincide, but at later times the more general form depicted may occur. Values of $u, v, w, \alpha, \beta, \epsilon, T, x$, and $t$ at point 6 and $w, \alpha, \beta, \epsilon$, and $T$ at point 7 are required. The large set of equations involved is most conveniently solved by an iterative process in which it is assumed initially that the value of $w$ at point 7 is equal to the value of $w$ at point 1 . This enables $\epsilon$, and $T$ at point 7 to be calculated using eq (28) along the time axis $(1,7)$ and eq $(27)$ aiong characteristic $(4,7)$ (segment $(6,7)$ in subsequent iterations).

The transverse wave front characteristic is constructed with slope $1 / P$ evaluated from point 2 data. The vertical line $(3,6)$ and the characteristic $(5,6)$ are then constructed and values of $w, \epsilon$, and $T$ at points 3 and 5 determined by interpolation. Values of $w, \epsilon$, and $T$ are calculated at point 6 using eq (26) along $(5,6)$, eq (27) along $(4,6)$, and eq $(28)$ along $(3,6)$. Values of $u, v, \alpha$, and $\beta$ are found using eq (36) along the transverse wave front characteristic $(2,6)$ and the compatibility relations (32) and (33) plus eq (7) applied at point 6 . Characteristic $(7,8)$ is then constructed with slope $(-1 / P)$ evaluated from point 7 data. The values of $\alpha$ and $\beta$ at point 7 are found by applying eq (37) along $(7,8)$ and eq $(7)$ at point 7 . The iteration cycle is completed by finding $w$ at point 7 through use of eq (9) along line segment $(6,7)$. Improved values of the required quantities are obtained by iterations which are continued until successive values of $w$ at point 7 are found to differ by a suffi- 
ciently small amount. During the calculation subiterations of the type discussed for situation $2 c$ are also carried out.

Figure $2 \mathrm{~b}$ depicts the situation encountered in tracing out the course of the transverse wave front characteristic; values of $u, v, w, \alpha, \beta, \epsilon, T, x$, and $t$ are required at point 7 . In this case values of $w, \epsilon$, and $T$ are found from relations holding along $(6,7)$ and the two constructed lines $(2,7)$ and $(5,7)$. Values of $u, v, \alpha$, and $\beta$ are found using eq $(36)$ along $(3,7)$ and applying the compatibility relations (32) and (33) plus eq (7) at point 7.

Situation $2 d$ occurs when values of $u, v, \alpha$, and $\beta$ at point 6 are calculated from data known at points 1,3 , and 5 , and from values of $w, \epsilon$, and $T$ at point 6 . The characteristic between points 3 and 5 may be either a $c$ characteristic or the transverse wave front characteristic. In this case, eqs (36) and (37) are applied respectively along the constructed $P$ characteristics $(2,6)$ and $(4,6)$, eq (9) is applied along the $c$ characteristic $(5,6)$, and eq $(7)$ is applied at point 6.

In situation $2 e$ values of $w, \alpha, \beta, \epsilon$, and $T$ at point 4 on the time axis are calculated from data at points 1 and 3 on a $c$ characteristic. In the solution of this problem, eq (28) is applied along the time axis, eq (37) along the constructed $P$ characteristic $(2,4)$, eqs $(9)$ and $(27)$ along the $c$ characteristic $(3,4)$, and eq $(7)$ at point 4.

In order to find the configuration of the filament in laboratory $\left(x^{\prime}, y^{\prime}\right)$ coordinates, it is desirable to know the values of $\xi$ and $\eta$ at each of the mesh points in the Lagrange diagram. Along the longitudinal wave front the values of $\xi$ and $\eta$ are zero, and along the transverse wave front the value of $\eta$ is zero. The value of $\xi$ is continuous across the transverse wave front. In region 1 the value of $\eta$ is zero and the value of $\xi$ is found in terms of $w$ and $\epsilon$ by integrating along a negative $c$ characteristic using the relation

$$
\frac{d \xi}{d t}=\frac{\partial \xi}{\partial t}+\frac{d x}{d t} \frac{\partial \xi}{\partial x}
$$

which becomes, after appropriate substitutions

$$
d \xi=(w-c \epsilon) d t
$$

In region $2, \xi$ and $\eta$ are found by integrating along a negative $c$ characteristic using the relations

$$
\begin{aligned}
& d \xi=[u-c(\alpha-1)] d t \\
& d \eta=(u-c \beta) d t .
\end{aligned}
$$

\section{Errors in a Previous Solution}

The scheme just presented has been outlined in more than the usual detail because it avoids several errors occurring in a previous solution [11]. Most of these errors were due to improper use of eq (9). It should be noted that eq (9), upon substitution of the boundary values for $u$ and $v$ at $x=0$, reduces to the relation $(\partial w / \partial t)_{x=0}=0$. This implies erroneously that $w$ is constant along the time axis. The mistake occurs because in this application of eq (9) $w$ has been expressed relative to a coordinate system fixed to the string at the point of impact, but the value of $w$ desired is that relative to a system fixed to the string at points in advance of the strain wave front. A similar error results if eq (9) is applied along the transverse wave front characteristic.

Another erroneous result is obtained if, in situation $2 e$, eq (9) is applied along the negative $P$ characteristic to find the value of $w$ at point 4 . In this example the value of $w$ at point 2 is obtained by interpolation between values at points 1 and 3 . Thus, the relation (9) is split into two components one of which is applied along the $t$ axis. situation $2 a$ provides a similar opportunity to make this mistake. 
Equation (7) can be expressed in the differential form

$$
\alpha d \alpha+\beta d \beta=(1+\epsilon) d \epsilon
$$

which is more convenient for calculation. Its use in this form, however, is unnecessary, and produces a small cumulative error.

If eqs (36) and (37) are combined with eqs (9) and (45), the following relations are obtained along the " $+P$ " characteristics:

$$
\begin{aligned}
& d u-P d \alpha=\frac{\alpha}{1+\epsilon}(d w-P d \epsilon) \\
& d v-P d \beta=\frac{\beta}{1+\epsilon}(d w-P d \epsilon)
\end{aligned}
$$

and along the " $-P$ " characteristics:

$$
\begin{aligned}
& d u+P d \alpha=\frac{\alpha}{1+\epsilon}(d w+P d \epsilon) \\
& d v+P d \beta=\frac{\beta}{1+\epsilon}(d w+P d \epsilon) .
\end{aligned}
$$

However, these relations, used previously [11], have yielded inaccurate and often erroneous results. They are, for instance, invalid along the transverse wave front characteristic when used in connection with eq (7) or (45), and invalid along the " $-P$ " characteristic in situations $2 a$ and $2 e$. In situation $2 d$ eqs (46) through (49) have yielded inaccurate results, especially in those meshes involving the transverse wave front characteristic.

\section{Coupling Between Longitudinal and Transverse Waves}

It is interesting to note that of the two wave phenomena present, the longitudinal wave is described in terms of the quantities $\epsilon, w$, and $T$, and the transverse wave in terms of the quantities $u, v, \alpha$, and $\beta$. It has been shown that the differential equations describing the problem can be reduced to a set involving $w, \epsilon$, and $T$ only; thus, it would seem that values of $w, \epsilon$, and $T$ could be found separately, and in a second step values of $u, v, \alpha$, and $\beta$ found in terms of them. Unfortunately, however, the boundary conditions are expressed in terms of the variables $u$ and $v$ so that in general this decoupling is impossible. ${ }^{4}$

From a physical point of view the effects of coupling between the variables describing the the longitudinal and transverse waves can be described as follows: Changes in the strain, tension, and particle velocity distributions due to viscoelasticity induce changes in the configuration and propagation velocity of the transverse wave which react back to modify the strain, tension, and particle velocity distributions. Thus, the viscoelastic effects can be considered as both primary and secondary.

The distributions resulting from the primary effect, are the same as those resulting from a longitudinal impact at constant velocity, in which the strain increases, the tension decreases, and the particle velocity stays constant at the point of impact. In the secondary or coupling effect the particle velocity at the point of impact changes with time, and associated changes occur in the strain, tension, and particle velocity distributions. If viscoelasticity is absent, as when the constitutive equation is of form $T=T(\epsilon)$, and if transverse impact occurs at constant velocity, only the primary effect is involved, and no changes occur in the longitudinal and transverse waves. In this case the problem may be considered as effectively decoupled.

${ }^{4}$ In the previous paper [11] eq (9) was applied along the $t$ axis and the erroneous result $u=a$ constant used as a boundary condition to achieve decoupling. 


\section{Implicit Method of Solution}

In the method of solution discussed above, explicit systems of equations are used to evaluate the unknowns at successive points in a characteristics network. Alternatively, values of the unknowns at points, along the string can be calculated simultaneously by the following algorithm, based on an implicit finite-difference formulation for nonlinear wave propagation problems [13].

In order to simplify the calculation, the mathematical problem is reformulated in terms of the five unknowns $u, v, \alpha, \beta$, and $T$. The two additional unknowns, $\epsilon$ and $w$, are defined by eqs (7) and (9), and can be found separately. The equations are the same as those used in the first method; namely, eqs (26) and (27) holding along the $\pm c$ characteristics, the constitutive eq (28), and eqs (36) and (37) holding along the $\pm P$ characteristics, except that the variables $\epsilon$ and $w$ have been evaluated in terms of the variables $u, v, \alpha$, and $\beta$, and do not appear.

The mesh system used is depicted in figure 3 . As before, there are three regions of interest: region 0 for points in advance of the longitudinal wave front, region 1 for the portion of the longitudinal wave in advance of the transverse wave front, and region 2 for points within the transverse wave. The differential equations have different forms in regions 1 and 2 . In region 1 there are no $P$ characteristics and the variables $v$ and $\beta$ are both equal to zero; thus only eqs (26), (27), and (28) with $\alpha-1$ substituted for $\epsilon$ and $u$ substituted for $w$ are used here. The more general forms of the equations with $\sqrt{\alpha^{2}+\beta^{2}}-1$ substituted for $\epsilon$ and $(\alpha d u+\beta d v) / \sqrt{\alpha^{2}+\beta^{2}}$ substituted for $d w$ are used in region 2. Along the longitudinal wave front the values of $u, \alpha$, and $T$ are found from eqs (29), (30), and (31) by substituting $\alpha-1$ for $\epsilon$ and $u$ for $w$, and along the $t$ axis the values of $u$ and $v$ are given by the boundary condition (16). Along the transverse wave front characteristic four compatibility conditions are needed. These are:

$$
\begin{aligned}
u_{2} & =u_{1}-P\left(\alpha_{2}-\alpha_{1}\right) \\
v_{2} & =-P \beta_{2} \\
T_{2} & =T_{1} \\
\alpha_{2}^{2}+\beta_{2}^{2} & =\alpha_{1}^{2}
\end{aligned}
$$

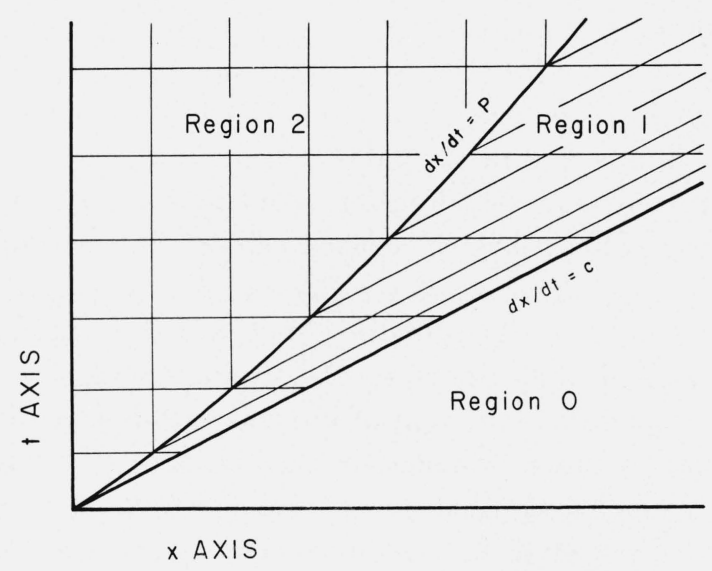

FIGURE 3. Mesh system used in the implicit method of solution.

The $\Delta x$ increments of the mesh are constant in region 2. The $\Delta x$ increments in region 1 and $\Delta t$ increments in regions 1 and 2 are variable, depending on the value of the velocity $P$ at the transverse wave front. 
where the subscripts refer to values of the variables in the regions 1 and 2. Equations (50) and (51) are the same as the compatibility conditions (20) and (21), and eqs (52) and (53) merely state mathematically that the tension and strain are continuous across the transverse wave front as was shown earlier in the text. Alternatively, eqs (52) and (53) may be obtained by equating horizontal and vertical components of the force across the transverse wavefront [3] or by the matrix methods expounded by Jeffrey and Taniuti [14].

In the mesh system the distance increment $\Delta x$ is maintained constant in region 2 , and a variable time increment is defined as $\Delta t=\Delta x / P$ where $P$ is the transverse wave front velocity averaged between points $(x, t)$ and $(x+\Delta x, t+\Delta t)$. In region 1 the distance increment depends on the value of $P$ and thus is variable also. The value of $P$ as a function of $x$ and $t$ is not known in advance, so the mesh system can only be constructed in stages as the calculation proceeds.

The calculation is carried out in a series of timewise steps in which the values of $u, v, \alpha, \beta$, and $T$ at each mesh point corresponding to $t+\Delta t$, are found in terms of values of these quantities known at each mesh point corresponding to time $t$. In the calculation each of the timewise steps is iterated several times in order that the trajectory of the transverse wave front (and value of $P$ ) be known with sufficient accuracy.

Two types of calculation, represented schematically in figure 4 , occur in each step. The simplest of these, figure $4 \mathrm{a}$, is used in region 1 to calculate values of $u, \alpha$, and $T$ at point 6 in terms of values known at points 1,2 , and 5 . In this calculation vertical line $(3,6)$ and line $(4,6)$ of slope $(-1 / c)$ are constructed, and the values of $u, \alpha$, and $T$ at points 3 and 4 determined by interpolation. The required values at point 6 are found by solving the equations holding along the characteristics $(1,6),(3,6)$, and $(4,6)$. The calculation is applied sequentially starting at the longitudinal wave front characteristic and working towards the transverse wave front characteristic.

The subcalculation, figure $4 \mathrm{~b}$, is used to find values of $u_{1}, u_{2}, v_{1}, v_{2}, \alpha_{1}, \alpha_{2}$, and $T$ at point $a$ on the transverse wave front and values of $u, \alpha$, and $T$ at the mesh points in region 2 corresponding to time $t+\Delta t$. The subscripts refer to regions 1 and 2 separated by the transverse wave front characteristic. For the seven unknown quantities at point $a$ there are seven corresponding equations, four holding along the characteristics $(b, a),(c, a),(d, a)$, and $(e, a)$ plus the three compatibility conditions (50), (51), and (53). Values of $u, v, \alpha, \beta$, and $T$ are not known at point $e$ however, so the values required at point $a$ cannot be determined explicitly by solving these seven equations. To find the five values required at point $e$ it is necessary to know the corresponding values at point $f$, and this requires the introduction of five equations holding along the $c$ characteristics $(g, f)$ and $(j, f)$, the $P$ characteristics $(h, f)$ and $(i, f)$, and the line $(d, f)$. Similarly, additional equations are required for each of the mesh points in region 2. The final point $p$ lies on the $t$ axis and is evaluated from three equations holding along $(q, p),(r, p)$, and $(s, p)$ plus the boundary conditions $u=0$, and $v=V$. In this way a large system of simultaneous equations is obtained, the solution of which gives the required values at each of the mesh points.

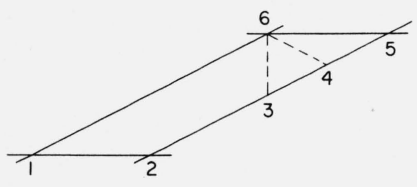

a.

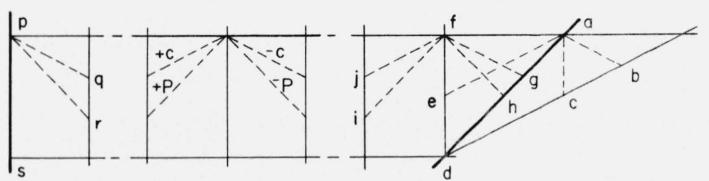

b.

Figure 4. Mesh situations encountered in the implicit method of solution 
This alternate formulation has several advantages. A fundamental set of equations is used in which the number of unknowns is equal to the number of relations holding along the characteristics. This set of five equations in $u, v, \alpha, \beta$, and $T$ is well posed for the prescribed initial and boundary conditions. Moreover, implicit schemes of this type are known to be stable at large values of time. Ordinarily, implicit schemes do not require small time steps in regions where the characteristics are nonlinear, but in this application the presence of a moving boundary of unknown curvature (transverse wave front characteristic) may limit this latter advantage.

\section{Calculated Results}

The explicit method has been used to calculate the behavior of the viscoelastic string for various values of the parameters $\lambda, m, c$, and $\tau$ characterizing the model and for various values of the transverse impact velocity $V$. The results for one of these calculations with parameters $\lambda=0.2$, $m=100 \mathrm{gm}^{-1}, \tau=0.002 \mathrm{~s}, c=100 \mathrm{~ms}^{-1}$, and $V=50 \mathrm{~ms}^{-1}$ are presented here. The values chosen for the parameters roughly simulate the material properties of an elastomer such as rubber when subjected to transverse impact.

The distributions of the particle velocity, strain, and tension calculated in this example are shown in figures 5,6 , and 7 , respectively. The ordinate in figure 5 is a dimensionless quantity, the ratio of the particle velocity at a Lagrangian distance $x$ along the string to the particle velocity at the instant of impact, $w / w_{0}$. The distribution of particle velocity in the wave is shown by the solid line curves for times of $1,2,3,4$, and $5 \mathrm{~ms}$ after impact. The dashed lines indicate the velocity distribution for the case of no relaxation $(\lambda=0)$. The distributions are similar to those resulting from longitudinal impact at constant velocity [10] except that in this example the particle velocity at the origin increases with time slightly, whereas in the case of longitudinal impact it necessarily remains constant. This illustrates the effect of coupling between the longitudinal and transverse waves when viscoelasticity is present.

The dimensionless ordinate in figure 6 is the ratio of the strain at a distance $x$ to the strain at the instant of impact, $\epsilon / \epsilon_{0}$. The distributions depicted are similar to those resulting from longitudinal impact at constant velocity. The strain at the point of impact increases with time and approaches a limiting value, but this limiting value is greater than the value of $\epsilon_{0}(1-\lambda)^{-1 / 2}=1.118 \epsilon_{0}$ which is

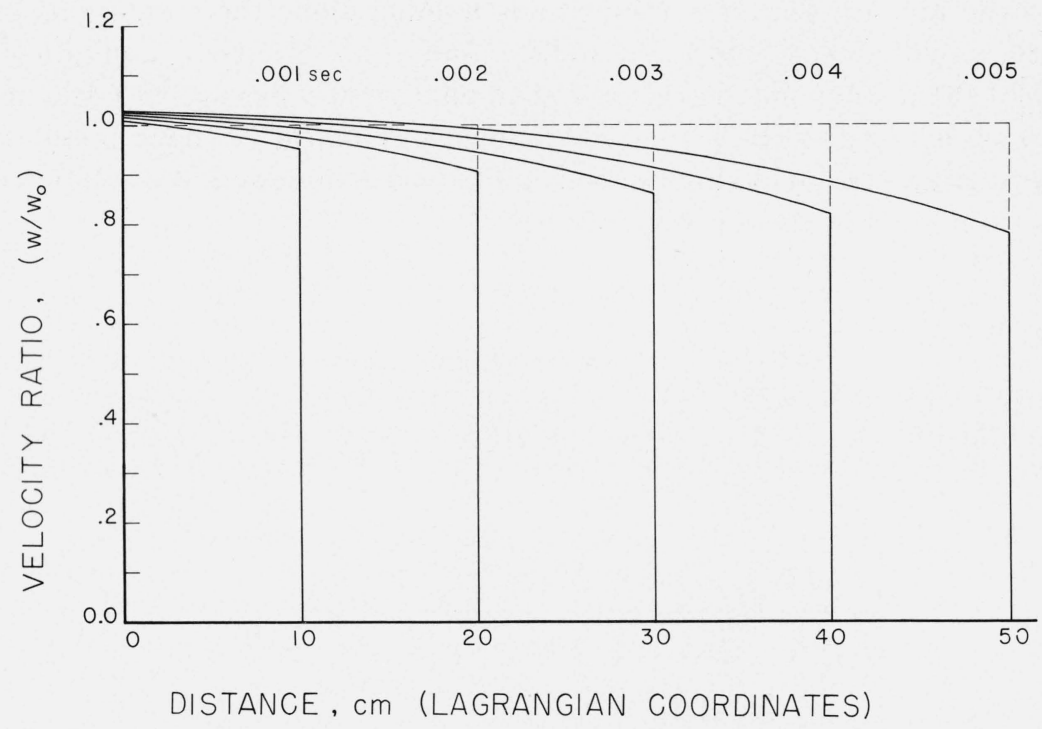

FigURE 5. Distribution of particle or flow-velocity along the string for times of $1,2,3,4$, and $5 \times 10^{-3} \mathrm{~s}$ after transverse impact.

Calculation parameters are $\lambda=0.2, m=100 \mathrm{gm}^{-1}, c=100 \mathrm{~ms}^{-1}, \tau=0.002 \mathrm{~s}$, and $V=50 \mathrm{~ms}^{-1}$. Dash lines give distributions for an elastic string $(\lambda=0)$. The particle velocity is expressed as the ratio of the velocity $w$ at distance $x$ to the velocity $w_{0}$ attained at the instant of impact. 


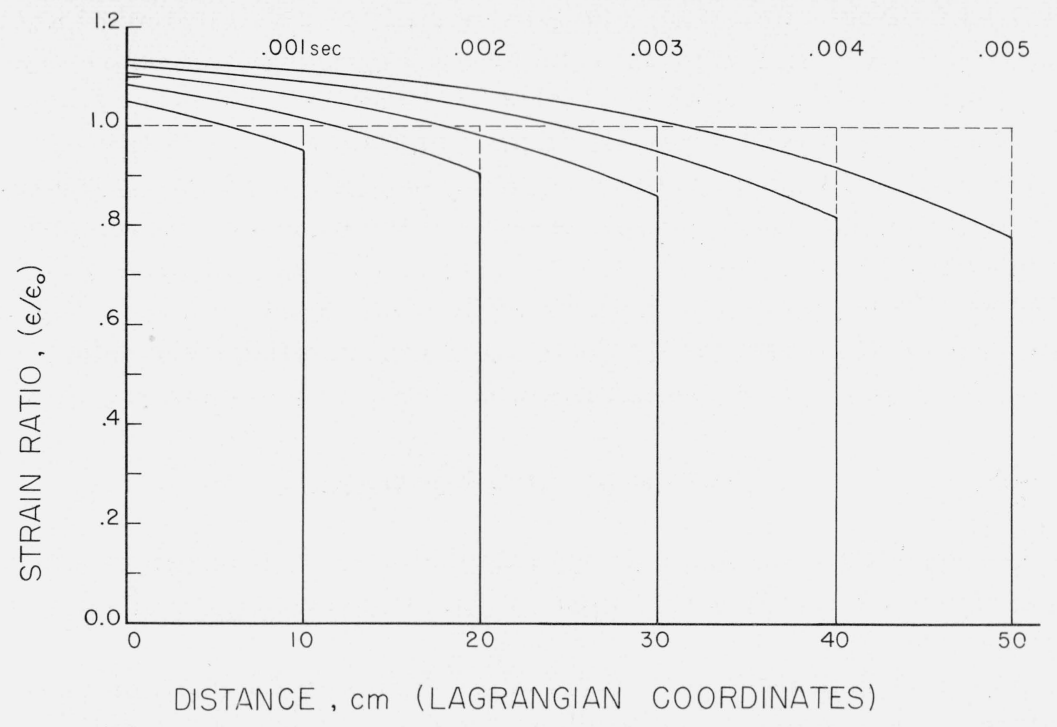

FIGURE 6. Distribution of strain along the string for times of $1,2,3,4$, and $5 \times 10^{-3} \mathrm{~s}$ after transverse impact.

Calculation parameters are $\lambda=0.2, m=100 \mathrm{gm}^{-1}, c=100 \mathrm{~ms}^{-1}, \tau=0.002 \mathrm{~s}$, and $V=50 \mathrm{~ms}^{-1}$. Dash lines give distributions for an elastic string $(\lambda=0)$. The strain is expressed as the ratio of the strain $\epsilon$ at distance $x$ to the strain $\epsilon_{0}$ attained at the instant of impact.

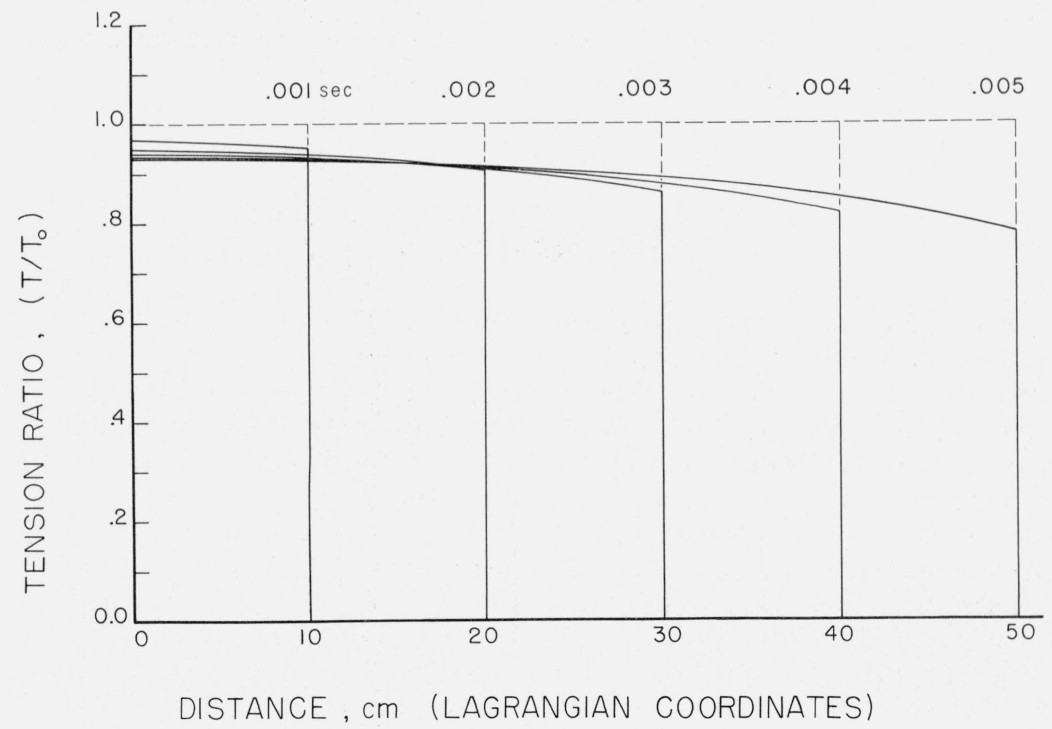

FIGURE 7. Distribution of tension along the string for times of $1,2,3,4$, and $5 \times 10^{-3} \mathrm{~s}$ after transverse impact.

Calculation parameters are $\lambda=0.2, m=100 \mathrm{gm}^{-1}, c=100 \mathrm{~ms}^{-1}, \tau=0.002 \mathrm{~s}$, and $V=50 \mathrm{~ms}^{-1}$. Dash lines give Calculation parameters are $\lambda=0.2, m=100 \mathrm{gm}^{-1}, c=100 \mathrm{~ms}^{-1}, \tau=0.002 \mathrm{~s}$, and $V=50 \mathrm{~ms}^{-1}$. Dash lines give
distributions for an elastic string $(\lambda=0)$. The tension is expressed as the ratio of the tension $T$ at distance $x$ to the tension $T_{0}$ attained at the instant of impact.

attained in the case of longitudinal impact [10]. The tension distributions at various times after impact are depicted in figure 7 . These distributions also are similar to those resulting from longitudinal impact at constant velocity. The tension at the point of impact decreases with time and approaches a limiting value which is less than the value of $T_{0}(1-\lambda)^{1 / 2}=0.894 T_{0}$ attained in the case of longitudinal impact. 
The values of particle velocity, strain, and tension attained at the instant of impact are -27.50 $\mathrm{ms}^{-1}, 0.2750$, and $2.750 \times 10^{2} \mathrm{~N}$. The initial value of the Lagrangian transverse wave front velocity is $46.44 \mathrm{~ms}^{-1}$ but after $5 \times 10^{-3} \mathrm{~s}$ it slows to $44.00 \mathrm{~ms}^{-1}$. To an observer in the laboratory $\left(x^{\prime}, y^{\prime}\right.$ system) the initial velocity is $\left(1+\epsilon_{0}\right) P_{0}+w_{0}=31.71 \mathrm{~ms}^{-1}$ and after $5 \times 10^{-3} \mathrm{~s}$ the wave front has traveled a distance $x^{\prime}=x+\xi=0.1525 \mathrm{~m}$. If the wave front had traveled at the initial velocity, it would have gone $0.1586 \mathrm{~m}$. At the instant of impact the slope of the transverse wave, $\tan \theta=\beta / \alpha$, is equal to -1.577 , but after $5 \times 10^{-3} \mathrm{~s}$ it decreases to -1.646 at the point of impact and -1.627 at the transverse wave front. Thus, the wave slows down as it propagates and gets slightly steeper and becomes slightly curved. However, for this calculated example these secondary coupling effects are small and would not be easily discerned by an observer in the laboratory.

\section{Summary and Conclusions}

This paper has described two methods for calculating wave propagation effects in a linearly viscoelastic string subjected to transverse impact at constant velocity. Results have been presented for a calculated example.

Viscoelasticity exerts both primary and secondary effects on the distributions of stress, strain, and particle or flow velocity along the string that result from constant-velocity transverse impact. The distributions resulting from the primary effect are the same as those resulting from a longitudinal impact at constant velocity, in which the strain increases, the tension decreases, and the particle velocity stays constant at the point of impact. In the secondary or coupling effect viscoelastic relaxation causes the particle velocity at the point of impact to increase with time, and associated changes to occur in the strain, tension, and particle velocity distributions.

The secondary effect also causes the transverse wave front to propagate at a gradually decreasing velocity. In addition, the transverse wave profile gets steeper and becomes gradually curved.

\section{References}

[1] Cristescu, N., Archiwum Mechaniki Stosowanej 12, 597 (1960).

[2] Cristescu, N., J. Mech. Phys. Solids 9, 165 (1961).

[3] Smith, J. C., McCrackin, F. L., and Schiefer, H. F., J. Res. NBS 60, 517 (1958); Textile Res. J. 28, 288 (1958).

[4] Schultz, A. B., Tuschak, P. A., and Vicario, A. A., Jr., J. Appl. Mech. 34, Trans. ASME 89, Series E, 392 (1967).

[5] Petterson, D. R., Stewart, G. M., Odell, F. A., and Mayheux, R. C., Textile Res. J. 30, 411 (1960).

[6] Petterson, D. R., and Stewart, G. M., Textile Res. J. 30, 422 (1960).

[7] Smith, J. C., Fenstermaker, C. A., and Shouse, P. J., Textile Res. J. 35, 743 (1965).

[8] Smith, J. C., and Fenstermaker, C. A., J. Appl. Phys. 38, 4218 (1967).

[9] Morrison, J. A., Quart. Appl. Math. 14, 153 (1956).

[10] Smith, J. C., J. Appl. Phys. 37, 1697 (1966).

[11] Smith, J. C., J. Res. NBS 70B, 257 (1966).

[12] Truesdell, C., and Toupin, R. A., The Classical Field Theories. Encyclopedia of Physics, edited by S. Flugge, Vol. III/1. (Springer-Verlag, 1960).

[13] Fong, J. T., Ph. D. Thesis, Stanford University (1966).

[14] Jeffrey, A., and Taniuti, T., Non-linear Wave Propagation with Applications to Physics and Magnetohydrodynamics (Academic Press, 1964).

(Paper 72B3-270) 